\title{
«Sur la phonologie de la phrase » de Serge Karcevski. Essai de relecture
}

\author{
D’Ottavi, Giuseppe \\ Institut des Textes et Manuscrits modernes, Paris (ITEM, UMR 8132 CNRS/ENS) \\ giuseppe.dottavi@ens.fr \\ Fougeron, Irina \\ Université Charles de Gaulle - Lille 3 \\ i.fougeron@,orange.fr
}

\begin{abstract}
Résumé. La place liminale, à la frontière entre Cercle pragois, École de Genève et Cercle de Moscou, que l'historiographie linguistique réserve à la figure de Serge Karcevski (1884-1955) a fini par laisser dans l'ombre l'apport spécifique de son œuvre et ses traits d'originalité. Une tension épistémologique très précise et très marquée ressort de l'ensemble de ses recherches : on y retrouve les questions de base de la linguistique théorique de son temps, qui portent sur la nature des unités minimes de la langue, sur le statut de la phrase et son rapport avec la notion de mot. D'un côté, le programme de recherche de Karcevski sont vigoureusement et explicitement généralistes (en témoigne le choix de rédiger en français ses travaux les plus accomplis), sa langue maternelle n'étant que le tout premier appui pour le développement d'une réflexion plus ample. De l'autre, son œuvre, traversée par plusieurs pôles d'intérêt (sémiologie, grammaire, syntaxe, non moins que la didactique), est régie pour autant par une batterie de notions-clés très caractéristiques : le langage comme lieu d'interaction de forces opposées se produisant dans un «équilibre instable », la langue comme lieu de la productivité ayant le dialogue comme centre. En dépit de son présumé rôle d'intermédiaire, Karcevski fait preuve d'une attitude remarquablement cohérente et novatrice : moins intéressé que les autres « russes » du Cercle de Prague à la recherche strictement phonologique, il s'engage dans le développement d'une phonologie à lui, d'inspiration typologique, portant sur la définition des schémas intonatifs comme invariants linguistiques. La méthodologie et les outils conceptuels mis en œuvre au cours de cette entreprise phonologique toute particulière font l'objet du présent essai. Notamment, notre propos majeur est de mettre en lumière les articulations principales de «Sur la phonologie de la phrase » (1931), le travail dans lequel cette recherche fait son apparition la plus accomplie.
\end{abstract}

\footnotetext{
Abstract. The liminal place, at the border between the Prague Circle, the Geneva School and the Moscow Circle, that the historiography of linguistics reserves to Serge Karcevski (1884-1955) eventually overshadows the real outcome of his work and its originality. A precise and strong epistemological tension is apparent from his whole research, which addresses the basic issues of the theoretical linguistic questions of his time, such as the nature of the fundamental units of language, the status of the sentence and its relation to the notion of word. On one hand, the research programme of Karcevski is made up of an explicit general linguistic outlook (the choice to write in French his most accomplished works confirms it), his mother tongue being just the first platform to start developing a broader investigation on language. On the other, his work, crossing over several domains (semiotics, syntax, even foreign language didactics), is governed by a battery of very characteristic key concepts: language as the place where opposing forces meet in an "unstable equilibrium", having the dialogue as his center. Despite his alleged role as an intermediary, Karcevski demonstrated a remarkably coherent and innovative attitude :
} 


\begin{abstract}
less interested than the other "Russians" of the Prague Circle to strictly phonological research, he engages himself in the development of a phonology theory on his own. Founded on typological bases, his phonology research aims at the definition of intonation patterns as linguistic invariants. The methodology and conceptual tools worked out in this specific phonological ground are the subject of this essay. Notably, our main purpose is to highlight the major junctions of his article "Sur la phonologie de la phrase" (1931) ("On the phonology of the phrase"), the work in which his phonological research appears in the most accomplished form.
\end{abstract}

\title{
1 Avant-propos
}

La place liminale, à la frontière entre Cercle pragois, École de Genève et Cercle de Moscou, que l'historiographie linguistique réserve à la figure de Serge (Sergej Osipovič) Karcevski(j) (1884-1955) a fini par laisser dans l'ombre l'apport spécifique de son œuvre et ses traits d'originalité.

Coincé entre des géants - V. Mathesius (1882-1945), N.S. Troubetzkoy (1890-1938), R.O. Jakobson (1896-1982) d'un côté, Ch. Bally (1865-1947) de l'autre -, mentionné comme l'un des signataires de la toute première ébauche des Thèses pragoises (1927) et à la fois comme inspirateur de la Société linguistique de Genève (1940), Karcevski n'est le plus souvent rappelé que pour son rôle de passeur des idées saussuriennes vers le Cercle de Prague ${ }^{1}$. Encore, parmi les raisons de la diffusion limitée de son œuvre - qui d'ailleurs ne se fait pas remarquer par son ampleur ${ }^{2}$ - on a évoqué la spécificité de son domaine de prédilection : la langue russe.

En réalité, d'un côté l'attitude et le programme de recherche de Karcevski sont vigoureusement et explicitement généralistes (en témoigne le choix de rédiger en français ses travaux les plus accomplis), sa langue maternelle n'étant que le tout premier appui pour le développement d'une réflexion assez large, qui touche plusieurs domaines de la linguistique. De l'autre, son œuvre, traversée par plusieurs pôles d'intérêt (sémiologie, grammaire, syntaxe, non moins que la didactique) est régie pour autant par une batterie de notions-clés très caractéristiques : le langage comme lieu d'interaction de forces opposées se produisant dans un « équilibre instable $3^{3}$ ", la langue comme lieu de la productivité ayant le dialogue comme centre.

En d'autres termes, une tension épistémologique très précise et très marquée ressort de l'œuvre de Karcevski dans son ensemble : on y retrouve les questions de base de la linguistique théorique de son temps qui portent sur la nature des unités minimes de la langue, sur le statut de la phrase et son rapport avec la notion de mot. En dépit de son présumé rôle d'intermédiaire, Karcevski fait preuve d'une attitude remarquablement originale : moins intéressé que les autres « russes » du Cercle de Prague à la recherche strictement phonologique, il s'occupe de développer une phonologie à lui, d'inspiration typologique, portant sur la définition des schémas intonatifs en tant qu'invariants linguistiques.

La méthodologie et les outils conceptuels mis en œuvre au cours de cette entreprise phonologique toute particulière font l'objet du présent essai. Notamment, notre propos majeur est de mettre en lumière les articulations principales de «Sur la phonologie de la phrase » (1931), le travail dans lequel cette recherche fait son apparition la plus accomplie.

L'œuvre de Karcevski s'insère de manière assez caractéristique dans les grands thèmes qui ont marqués les recherches des Pragois (phonologie, études grammaticales, poétique) et des Genevois (l'exploitation de la dialectique langue/parole déclinée autour de la notion de système) : notre essai se veut ainsi une 
tentative de garder l'orientation entre ce qui est pragois, ce qui est genevois, et - surtout - ce qui est, finalement, karcevskien.

\section{Brefs repères biographiques}

La vie de Karcevski se partage entre, au moins, deux patries ${ }^{4}$. Né à Tobolsk, en Sibérie, le 28 août 1884 , Karcevski y obtient son diplôme d'instituteur en 1903 et part enseigner à Nahrači (aujourd'hui Kondinskoje, en Sibérie occidentale). Membre du Parti socialiste-révolutionnaire - à l'époque l'un des concurrents principaux du parti bolchevique de Lénine -, en 1906 il est arrêté à Moscou et emprisonné. Après un an de détention, il réussit à s'évader et à gagner la Suisse, pour s'installer à Genève, où il s'inscrit à l'Université, suit les cours de F. de Saussure (1957-1913) et Ch. Bally (1965-1947), avant d'être licencié ès Lettres en $1914^{5}$.

Après la Révolution, en mars 1917 Karcevski rentre en Russie où il participe aux travaux du Cercle linguistique de Moscou, fondé par R. Jakobson et G.O. Vinokour (1896-1947) et affilié à la Commission de Dialectologie (rattachement de l'Académie des Sciences de Russie). C'est ainsi qu'il se lie avec les personnalités majeures de la linguistique russe de l'époque, tels que D.N.O. Ušakov (1873-1942), N.N. Durnovo (1876-1937) et surtout A.M. Peškovskij (1878-1933), dont les études exerceront une influence considérable sur son propre travail. C'est au cours des séances du Cercle linguistique de Moscou qu'il présente, en mars et mai 1918, les principes de sa propre classification du verbe russe. Ce matériau ne verra pourtant le jour qu'une dizaine d'années plus tard sous une forme homogène bien que mutilée (Karcevski 2004) ${ }^{6}$. C'est le chapitre d'introduction à cet ouvrage, écrit à nouveau à l'occasion de sa parution, qui constitue le premier essai entièrement et explicitement consacré aux questions de linguistique générale, qui est un précieux exemple d'une branche alternative de la tradition de l'héritage saussurien ${ }^{7}$.

En 1919, Karcevski quitte définitivement la Russie. Grâce à l'appui d'A. Meillet (1866-1936) - avec lequel il démarre un travail de thèse - l'Université de Strasbourg lui offre un poste de lecteur de russe. Deux ans plus tard, on le retrouve à Prague, où il rejoint Jakobson : les années 1920 marquent la participation active de Karcevski au sein du Cercle linguistique de Prague ${ }^{8}$.

Le 11 mai 1927, le Doyen de l'Université de Genève autorise l'impression de la Thèse de doctorat de Karcevski («Système du verbe Russe. Essai de linguistique synchronique »: Karcevski 2004), soutenue devant un jury composé de Ch. Bally et Max Niedermann (1874-1954). Finalement, à partir de l'année suivante, l'Université de Genève se l'attache en qualité de lecteur, puis de maître de conférences et, seulement après la Guerre, de professeur (extraordinaire en 1946, ordinaire en 1954) de Langues et littératures slaves. En 1928, à Moscou, paraît son Précis de langue russe [Povtoritel'nyj kurs russkogo jakyka].

Karcevski passera le reste de sa vie à Genève, où sous son impulsion - et sous la suggestion peut-être de l'expérience pragoise - est convoquée la première réunion de la future Société genevoise de linguistique. Le 4 mai 1940 il se charge d'en inaugurer les séances, avec une communication ayant pour titre «Deux propositions dans une seule phrase (chapitre de syntaxe russe) » ${ }^{9}$. En Suisse, cependant, il souffre d'isolement scientifique : ses travaux ne sont pas lus et les problèmes financiers l'empêchent de mener à bout ses recherches.

A la veille de son retour projeté en Russie, Karcevski s'éteint, à Genève, le 7 novembre 1955. Ses amis et collègues genevois lui rendent hommage avec un numéro des Cahiers Ferdinand de Saussure (14, 1956) entièrement consacré à sa mémoire et qui contient l'importante nécrologie par Jakobson (1956) - un texte qui a largement contribué à répandre une certaine image du linguiste - ainsi que deux inédits (Karcevski 1937b; 1940). L'année suivante, paraît l'article de N.S. Pospelov (1890-1984) qui marque la réintroduction de Karcevski dans le milieu scientifique russe, après les années de triomphe du marrisme et après la censure qui avait touché les membres du Cercle de Prague (condamné comme expression d'une « linguistique bourgeoise $\left.{ }^{10} »\right)$. 


\section{Vers une nouvelle approche de l'intonation}

Dans l'avant-propos de son Précis de la langue russe (1928) - qui reprend sous une forme plus accessible aux élèves de terminale (auxquels il est adressé explicitement) les positions illustrées dans sa Grammaire (1925) - Karcevski se donne comme but de produire « une description de notre langage en accord avec les bases linguistiques " [dat' opisanie elementarno naučnoe]. Un tel accord est réalisé de manière subversive ainsi que didactiquement efficace : le chapitre portant sur la phonétique et la phonologie du russe est repoussé à la fin de l'ouvrage, qui s'ouvre alors sur la «Syntaxe », et tout particulièrement sur les éléments de la construction de la phrase. Dans la présentation de sa propre langue, il faut suivre - soutient Karcevski - « la voix naturelle » qui procède des unités de sens vers les unités de son, et non pas l'inverse. Selon une démarche authentiquement saussurienne ${ }^{11}$, Karcevski considère que l'approche de la face phonique du langage dépourvue de la prise en compte des unités de sens qu'elle comporte nous conduit hors de la linguistique, vers d'autres sciences, telles que la physiologie des sons et l'acoustique (Karcevski $1928: 103)^{12}$.

Cette même attitude, comme nous venons de le rappeler, était en effet déjà présente dans sa Grammaire, dont l'ouverture fait pourtant preuve d'une démarche supplémentaire, instituant déjà un rapport direct entre syntaxe et intonation :

Notre langage est composé d'énoncés. À chaque énoncé plus ou moins terminé correspond une phrase. La phrase est composée de mots qui sont reliés les uns aux autres selon les lois de la grammaire et assemblés par l'intonation. (Karcevski 1925 : 12)

Karcevski met en avant l'idée selon laquelle le «mot» (résultat d'opérations surveillées par la « grammaire ») n'est qu'un terme virtuel ou « potentiel » de la phrase, une roue dans son mécanisme. Ce mécanisme fonctionne selon un critère syntagmatique : les membres qui s'associent en phrase sont liés syntagmatiquement c'est-à-dire en fonction de leur rôle en tant que déterminé $(\mathrm{T})$ et déterminant $(\mathrm{T}$ '). Si la phrase est l'unité première de communication ${ }^{13}$ et le syntagme comme structure binaire en fournit les constituants, c'est l'intonation qui gouverne l'assemblage. La notion d'intonation fait ici son entrée comme principe organisateur des unités constitutives de la phrase.

On pourra remarquer que, à l'époque, le phénomène de l'intonation constitue encore un domaine de frontière dans les études linguistiques, sinon phonétiques. La place qu'y consacre par exemple le Traité de M. Grammont est minime (1933: 125-135) ${ }^{14}$. Dans les premières décennies du XX ${ }^{\mathrm{e}}$ siècle, pour ne se borner qu'à l'Europe continentale, ce sont justement Bally et Mathesius qui encadrent le phénomène, affirmant sa nature de procédé «implicite » de l'énonciation (Bally 1965 [1932] : 47 et ss., 86 et ss. ; 1941) et, surtout, en explorant spécifiquement son rôle sémantique (Mathesius 1911, 1929). C'est tout particulièrement le travail de Mathesius qui fournit à Karcevski l'arrière-plan méthodologique déterminant : si la fonction de l'intonation est d'organiser l'information passée du parlant à l'écouteur en linéarisant la hiérarchie des structures syntaxiques (c'est-à-dire établir le lien entre le connu - « thème »et l'apport d'information nouvelle - « rhème »), il ne faut pas séparer le niveau sémantique du niveau contextuel de la communication ${ }^{15}$.

Comme Mathesius le raconte (1936: 144), une Réunion Phonologique Internationale est organisée à Prague entre le 18 et le 21 décembre 1930 dans le but de définir et mettre au point une position commune et « officielle» du Cercle de Prague vis-à-vis des questions phonologiques, considérées par ses membres comme leur propre «cri de guerre » (ibid.) à émettre pendant le Congrès international des Linguistes, prévu pour l'année suivante à Genève.

C'est dans le cadre de cette manifestation que Karcevski présente, le 19 décembre, dans l'après-midi, une communication portant sur la définition d'un nouveau champ de la phonologie. Les actes verront tout de suite le jour, réunis dans le quatrième volume des Travaux du Cercle de Prague (1931), à temps pour le Congrès de Genève : parmi eux « Sur la phonologie de la phrase » de Karcevski.

Tous les termes de l'intitulé de l'essai - qui dans son ensemble peut bien avoir (eu) un air provocateur demandent une explication. 
Le point de départ de l'argumentation de Karcevski (présenté par ailleurs dans sa forme la plus accomplie dans les premières pages de l'introduction au Système du verbe russe ${ }^{16}$ ) consiste en un geste de distinction et de définition. Il s'agit d'identifier, au sein d'une même langue, plusieurs «plans »: morphologie et phonologie (plans « phoniques ») d'un côté, lexicologie et syntagmatie ("plans conceptuels ») de l'autre. Ce dernier, à son tour, s'offre sous deux facettes : une « externe » (la syntaxe) et une « interne » (la dérivation). Voici le rapport qui relie, selon Karcevski, la morphologie à la syntaxe : tout ce qui sépare les deux domaines ne relève que de la frontière du « mot », c'est-à-dire ce n'est pas une question de substance ${ }^{17}$. C'est sur cette idée de co-extensivité substantielle de la morphologie et la syntaxe que se développe la réflexion de Karcevski sur l'intonation.

Tout comme la «phonologie » et la «syntaxe» ont subi un repositionnement à l'intérieur du cadre épistémologique commun, la définition de la «phrase » bouscule aussi. L'outil conceptuel exploité par Karcevski pour rendre raison de sa conception de la phrase est la notion d'actualisation. Concept éminemment sémiologique, l'actualisation est la dynamique qui relie le plan « virtuel» au plan « actuel» de la langue. Or, Bally - dont Karcevski se réclame - exploite amplement ce mécanisme, mais pour l'appliquer à un univers langagier structuré différemment. Pour Bally, en effet, la «phrase » - en tant que « la forme la plus simple de la communication de la pensée » (où "penser » c'est "réagir à une représentation en la constatant, en l'appréciant ou en la désirant », Bally $1965: 35$ ) - ne relève que du plan de la parole. Tout en mettant en œuvre ce même principe d'implémentation de la langue dans la parole, l'approche de Karcevski affiche, dès le départ, une attitude assez différente.

Karcevski - qui reprend, cette fois aussi, une intuition de Mathesius $(1911,1929)$ qui appelle « clause » le schéma grammatical susceptible d'être réalisé dans la parole - écrit :

La phrase est une unité de communication actualisée. Elle n'a pas de structure grammaticale propre. Mais elle possède une structure phonique particulière qui est son intonation. C'est précisément l'intonation qui fait la phrase (Karcevski 1931:89).

Suivant Karcevksi la "phrase » n'est pas tant l'unité dotée d'une cohérence ou cohésion grammaticale quelconque, que l'unité qui possède une intonation. En d'autres termes, la définition de phrase ne passe pas par la morphologie, ni par la syntaxe ou un autre critère formel : la phrase ne se donne que dans sa dimension énonciative, actuelle, singulière.

La position de Karcevski - pour lequel la phrase devient le lieu de la superposition de la composante phonético-intonative à la composante " grammaticale » - tire un trait de connexion très forte entre deux domaines différents, en liant l'un à l'autre à travers un rapport de hiérarchie, jusqu'alors inexploré en tant que tel. Le plan syntagmatique d'une expression langagière rejoint le plan prosodique :

la phonologie de la phrase [est] l'ensemble des phénomènes phoniques qui servent à distinguer la phrase des autres unités de langue, telles que le syntagme, le mot, etc. (Karcevski 1931: 88).

Ainsi, en choisissant d'appliquer à un nouvel objet le mot d'ordre de ses amis pragois (la phonétique envisagée sous la perspective d'une approche fonctionnelle), Karcevski se livre à une entreprise de fondation qui ouvre une voie toute nouvelle à la recherche phonologique.

\subsection{Les types d'intonation}

À une époque où, comme nous l'avons remarqué, les études sur l'intonation n'étaient pas particulièrement développées, Karcevski a pu se tourner vers la tradition russe, qui se présentait extraordinairement riche à ce sujet, se nourrissant bien des études des grammairiens, mais aussi des travaux de spécialistes du théâtre. Karcevski (1931) se sert à plusieurs reprises, par exemple, des études de A.M. Peškovski (1878-1933) - nombreux exemples sont tirés de la célèbre étude sur la syntaxe du russe (Peškovski 1928a) - et les recherches de V.N. Vsevolodskij-Gerngross (1882-1962) sont mentionnées également parmi ses sources principales ${ }^{18}$. 
En 1914 - lors de la parution de la première édition de son travail sur la syntaxe russe - Peškovski est le premier dans la littérature scientifique de son pays à introduire systématiquement la description des traits intonatifs (et la formalisation de plusieurs "schémas mélodiques ») dans le cadre d'une étude de linguistique $^{19}$. Ce qui intéresse Peškovski, c'est l'intonation comme indice du rôle syntaxique de la phrase : il considère notamment l'intonation comme l'un des moyens aptes à distinguer les rapports de coordination et de subordination ${ }^{20}$. Dans l'autre étude de Peškovski exploitée par Karcevski (1931 : 116), Intonacija i grammatika (Intonation et grammaire, 1928b), l'intonation du mot (ainsi que celle de la syllabe) n'est prise en compte que dans son essence phonético-physiologique. Quant à l'intonation de la phrase, elle est significative car elle est «symbolique » : un lien de proportionnalité directe s'établit entre la montée de la voix dans l'interrogation et l'interrogation dans la pensée.

Vsevolodskij-Gerngross - à peu-près le seul non linguiste mentionné par Karcevski ${ }^{21}$ - est l'auteur d'une Théorie de l'intonation du discours russe (1922), une étude dans laquelle le phénomène de l'intonation est pris en compte à partir de la prononciation «correcte et exemplaire » des acteurs. La variation de l'intonation est rapportée à la modification du ton qui touche la syllabe, considérée comme l'unité minimale de l'analyse. Suivant ce critère, les analyses des phrases par Vsevolodskij-Gerngross présentent le mouvement du ton d'une syllabe à l'autre (Vsevolodskij-Gerngross 1922 : 47-51). Il arrive ainsi que la notion d'intonation risque souvent, chez Vsevolodskij-Gerngross, d'être confondue avec celle de mélodie.

Après avoir passé en revue un très large nombre d'occurrences de phrases, littéraires et du parlé, tirées (ou traduites) du russe, du français, de l'italien, de l'allemand etc., Karcevski dégage deux grands types d'intonation : intonation tendue («anti-cadence ») et intonation relâchée (« cadence »).

Ces deux types d'intonation permettent la division de la phrase en constituants, c'est-à-dire en unités de sens disposées suivant leurs rôles relatifs dans l'ensemble. Des deux membres de phrase contigus c'est le premier qui porte la marque de leur reliement («anti-cadence »), le second ne manifeste d'aucune façon qu'il est rattaché à ce qui précède. Ainsi «le début de la phrase ou d'un membre de phrase n'a pas de valeur phonologique $^{22}$ ». Dans un débit rapide, la pause entre les membres de phrase peut ne pas se réaliser. C'est là qu'intervient la «mi-cadence $»^{23}$. À l'intérieur d'un membre de phrase, la «micadence » devient le signal d'une césure et d'une reprise immédiate, tout à la fois :

Peu nous importent les caractères phoniques exacts de la syllabe affectée par [la] micadence [...] l'essentiel est qu'en position de mi-cadence la syllabe a une physionomie particulière. (Karcevski 1931 : 97).

L'intonation possède un caractère progressif : les modifications réalisées annoncent les configurations des faits à venir. C'est ainsi la montée mélodique sur le thème d'une phrase assertive appelle une chute du ton sur le noyau du rhème. À l'inverse, et les analyses instrumentales récentes le montrent, un ton plat, monocorde dans le thème interrogatif laisse prévoir une montée dans le rhème, et par conséquent l'émission d'une interrogation.

Les unités de la phrase peuvent coïncider ou non avec les unités syntaxiques, du moment qu'il s'agit d'une segmentation d'un tout autre ordre. Entre les membres de la phrase il existe quatre espèces de rapports structuraux. À chaque type de rapport correspond un type d'intonation : 


\begin{tabular}{|c|c|c|c|}
\hline \multicolumn{2}{|c|}{$\begin{array}{l}\text { DIFFÉRENCIATION } \\
\text { QUALITATIVE }\end{array}$} & \multirow[t]{2}{*}{$\begin{array}{c}\text { RAPPORTS } \\
\text { STRUCTURAUX }\end{array}$} & \multirow[t]{2}{*}{ INTONATION } \\
\hline égalité & inégalité & & \\
\hline \multirow[t]{2}{*}{$\begin{array}{c}\text { identité } \\
\text { (série ouverte) } \\
\end{array}$} & & $\begin{array}{c}\text { répétition } \\
\text { (série ouverte d'identité) }\end{array}$ & intonation d'identité \\
\hline & $\begin{array}{c}\text { gradation } \\
\text { (série ouverte) } \\
\end{array}$ & $\begin{array}{c}\text { échelonnage } \\
\text { (série ouverte d'inégalité) }\end{array}$ & intonation graduée \\
\hline \multirow[t]{2}{*}{$\begin{array}{c}\text { contraste } \\
\text { (série fermée) } \\
\end{array}$} & & $\begin{array}{c}\text { symétrie } \\
\text { (égalité contrastante) }\end{array}$ & intonation de symétrie \\
\hline & $\begin{array}{c}\text { contraste } \\
\text { (série fermée) }\end{array}$ & $\begin{array}{c}\text { asymétrie } \\
\text { (contraste par inégalité) }\end{array}$ & $\begin{array}{c}\text { intonation d'asymétrie }= \\
\text { neutre }(\text { enclave }))\end{array}$ \\
\hline
\end{tabular}

Les intonations tendues peuvent se suivre, se succéder, alors que l'intonation relâchée ne marque, en règle générale qu'un seule membre. L'intonation la plus importante est celle de symétrie, celle qui permet la division en thème et rhème (le loup / est gris) ${ }^{24}$. Ensuite vient l'intonation graduée - sur la ligne sont disposées des parties de sens analogue ; la tension va toujours crescendo : je ne comprends pas / comment vous / si bon / pouvez agir ainsi et en plus vous en vanter. Puis vient l'intonation d'identité : reproduction exacte du membre précédent (énumération) : [Devant ses yeux] défilent des guérites / des paysannes /des gamins / des boutiques / des réverbères (A.S. Puškin, Evgenij Onegin). En dernier, vient l'intonation d'asymétrie — c'est l'intonation de l'enclave, des parenthèses, une intonation neutre à l'emploi très varié : demain / m'a dit mon frère / je dois partir.

L'attitude typologique de sa recherche se réalise dans cette systématisation: suivant Karcevski, elle s'applique à toute langue naturelle.

\section{Développements ultérieurs}

Les idées présentées dans «La phonologie de la phrase » trouvent un complément dans l'article «Phrase et proposition », qui paraît en 1937 dans les Mélanges J. van Ginneken (Karcevski 1937a).

Ici se trouve la formulation la plus claire concernant la nature de la phrase comme produit du dialogue. De plus : «La parole intérieure est, elle aussi, un dialogue mental » (ibid., p. 127) ${ }^{25}$. Le dialogue est envisagé comme l'alternance de question et de réponse, ou comme une discussion où s'affrontent des thèses opposées ou des énoncés différents. La question et la réponse se conditionnent réciproquement, une n'existe pas sans l'autre, tandis que l'énoncé (sauf dans le cas de l'énoncé négatif, qui suppose une affirmation préalable) peut être à lui seul un tout indépendant. L'intonation ici reflète l'opposition des deux protagonistes : l'un qui demande l'information et l'autre qui la possède. Ainsi, l'interrogation se manifeste en une tension, représentée par une mélodie montante, alors que la réponse apporte la détente, ce qui se manifeste par la cadence (l'intonation est calmante).

Dans l'énoncé la personne parlante réunit en soi-même le sujet questionnant et le sujet répondant. De la même manière, la courbe de l'intonation réunit la tension interrogative et la détente de la réponse. Ainsi l'opposition est surmontée, ce qui assure à l'énoncé une relative autonomie. Si la phrase s'organise à travers l'intonation, la proposition est faite par une certaine structure grammaticale et rien ne permet de prévoir dans quel type de phrase cette structure peut être actualisée. 
La centralité du rôle du dialogue dans l'exercice de la parole est reprise par Karcevski et affirmée à nouveau dans «Deux propositions dans une seule phrase » (Karcevski 1940), où le rapprochement de deux propositions se fait à l'image de la rencontre de deux répliques dans un dialogue :

ce sont les situations dialogales, les positions réciproques des partenaires qui déterminent la structure de la phrase en général et de la phrase complexe en particulier. La parole intérieure, elle aussi, imite la marche du dialogue. (Karcevski $1940: 39)$.

Le dialogue « informatif » (question - réponse) est à la base de la subordination, alors que le dialogue « oppositif » (échange de répliques) est à la base de la coordination. La position des protagonistes A et B n'est pas sans comporter un écart décisif : B est toujours avantagé, car il peut mettre fin au dialogue avec «le dernier $\operatorname{mot}^{26} »$.

La question de l'enchaînement des phrases, abordée par Karcevski (1931), trouve un développement conséquent dans un texte datant du 1942, mais dont la version française originale n'a été publiée qu'en 2000 : «Asyndète et subordination en russe » (Karcevski 1942). Les phrases enchaînées ne sont pas simplement juxtaposées. Elles sont, tout comme les membres qui constituent la phrase, enchaînées entre elles : lorsque la première phrase modifie sa cadence, une suite est attendue. On arrive ainsi à un groupe de phrases, a une unité supérieure qui est marquée par une vraie [hyper-]cadence. C'est justement cette notion de cadence « plus décisive » que Karcevski reprend dans son analyse de l'asyndète.

Psychologiquement (et historiquement) antérieure à la subordination, l'asyndète s'oppose à la coordination comme la non-explicitation des liens à l'explicitation. Les termes accouplés sont coordonnés au moyen de la conjonction zéro. Trois variétés de phrases asyndétiques sont dégagées :

1. La seconde proposition est simplement surajoutée à la première, constituée déjà en phrase.

La seconde proposition n'est pas prévue par le locuteur au moment où il énonce la première. Le locuteur ne s'aperçoit qu'après coup (lorsqu'une cadence a déjà fermé la communication) qu'il veut donner une suite à son énonciation. C'est là qu'il ajoute une nouvelle cadence, plus décisive encore que la première. Ainsi, une séquence progressive, ouverte, se réalise, sa caractéristique phonologique étant la présence d'une cadence à l'intérieur de la phrase.

2. Le second acte de prédication est prévu dès le départ et il est annoncé par le premier.

La seconde prédication se déduit ainsi de la première comme une prémisse. Une séquence régressive, fermée, se produit. L'absence de cadence à l'intérieur de la phrase est soulignée par une forte anticadence qui signale que la fin de la première proposition ne constitue pas la fin de l'acte de communication. L'intonation de la seconde partie est naturellement celle d'une détente.

Dans une rédaction manuscrite alternative, on lit :

Ces deux types de structures asyndétiques diffèrent comme séquences opposées. Dans le premier, de caractère analytique, la prédication principale forme la première partie de la phrase en en constituant un tout achevé, auquel la seconde prédication s'ajoute à la manière d'un appendice, ce qui est un rapport de pure relation. Dans l'autre type, par contre, la prédication principale est concentrée dans la seconde partie et se rattache à la première comme son prédicat. Il s'agit donc d'un rapport d'inhérence. Ce second type est synthétique. (Karcevski $1942: 234$ )

Aucun rapport d'énumération n'est impliqué dans ces deux types : l'énumération, qui comprend normalement plus de deux membres, n'établit, par définition, aucun rapport entre les unités contiguës qu'elle rassemble. Tout ce qui les rapproche, et l'intonation le fait bien ressortir, c'est l'identité de leur position vis-à-vis d'un terme commun mais étranger à la série.

3. Le troisième type d'asyndète est neutre du point de vue de la suite des séquences.

Ici, il s'agit de traduire une double aperception : l'intonation indique que l'incidente constitue un corps étranger par rapport au reste de la phrase. 


\title{
5 Conclusion
}

Au cours des mêmes années où Karcevski poursuit sa recherche, au sein de la tradition russe se développe une réflexion assez riche et originale autour du dialogue comme phénomène discursif fondamental. Cette tradition, commencée par l'étude dialectologique de Lev Ščerba (1915) et consolidée par l'essai remarquable de Lev Jakubinskij (1923) ${ }^{27}$, met en avant une conception du dialogue comme lieu d'invention, innovation et évolution linguistique, opposé au monologue comme état de langue à la fois fixe et factice, où se concentrent les produits de la dimension normative de l'usage :

\begin{abstract}
Le monologue est une forme artificielle [...] l'existence réelle de la langue se manifeste seulement dans le dialogue. C'est dans le dialogue que les mots nouveaux, formes et expressions nouvelles sont forgés ; on applique plutôt au dialogue l'effet des facteurs psychologiques et physiologiques, qui change la langue, et un chercheur qui veut étudier ces facteurs doit prendre en compte cette forme de manifestation de la langue. (Ščerba $1915: 4$; trad. Ivanova $2000: 118$ )
\end{abstract}

Telle dialectique (caractère naturel ou « réel » du dialogue versus caractère « artificiel » du monologue) qui a ses bases par ailleurs dans la thématisation de l'écart entre langue écrite et langue parlée - a nourri un vaste échange transdisciplinaire, engageant, au-delà de la linguistique, la stylistique (avec V.V. Vinogradov), la théorie littéraire (B.M. Eichenbaum), la philosophie (M.M. Bakhtine), la psychologie (L.S. Vygotskij) ${ }^{28}$.

Comme nous venons de le mettre en évidence, la notion de dialogue est également au centre de la conception linguistique générale de Karcevski, et pourtant aucune trace de ce débat ne se retrouve dans les écrits que nous avons considérés ici. Non seulement aucune mention des travaux fondateurs de Ščerba (1915), Jakubinskij (1923) ou Bakhtine (1929) par exemple n'est faite par Karcevski (1931; 1937a ; 1940), mais Karcevski semble ignorer de même les travaux de Boris M. Eichehnbaum (1886-1959), qui évoquent justement l'intonation jointe à la composante mimique et gestuelle de l'échange dialogal ${ }^{29}$.

Si d'un côté on peut tranquillement affirmer que Karcevski ignorait tout simplement ces branches d'études (Karcevski ne manque pas, par ailleurs, de se plaindre, dans sa correspondance privée, de son isolement scientifique), de l'autre on est porté plutôt à interpréter ce silence comme signe d'une attitude d'indépendance marquant la volonté de souligner l'originalité de son approche, en l'encadrant dans un véritable programme de fondation d'un domaine nouveau de la phonologie.

\section{Appendice. Un parallèle du côté de la philosophie du langage}

Sans qu'il soit possible établir des liens directs entre les deux ${ }^{30}$, certaines remarques portées sur le statut de la phrase par Ludwig Wittgenstein (1889-1951) offrent des parallèles suggestifs avec le rôle de l'intonation comme notion linguistico-épistémologique avancée par Karcevski.

Dans le cadre d'un examen critique de la notion de «proposition » mené notamment à propos de sa définition comme argument possible d'une fonction de vérité au sein d'un système linguistique donné ${ }^{31}$, Wittgenstein :

Quand nous nous interrogeons sur la forme générale de la proposition, il faut penser que dans le langage ordinaire les propositions ont certes un rythme et une sonorité déterminés, mais que nous n'appelons pas «proposition » tout ce qui sonne comme une proposition. - C'est pourquoi on parle de propositions sensées et de propositions dépourvues de sens. [...] Mais d'un autre côté cette résonance propositionnelle n'est pas essentielle à ce que nous appelons proposition en logique. [...]. Donc se pose la question : abstraction faite de cette résonance propositionnelle qui nous induit en erreur, avons-nous encore un concept général de la proposition? (Wittgenstein 1980 : 164 [I, § 78])

Plus avant dans le même texte, au cours d'une réflexion portée sur le fondement du mécanisme de la compréhension linguistique, c'était pourtant la «résonance propositionnelle » qui a attiré l'attention de 
Wittgenstein. La compréhension d'une proposition est apparentée justement à la perception musicale et le lien intime qui relie la proposition à un morceau de musique est évoqué :

\begin{abstract}
Le fait de comprendre une proposition est plus proche qu'on ne croirait de la compréhension d'un morceau de musique [...] (Souvenez-vous de ce qui se passe quand on lit une proposition avec une intonation inexacte, que de ce fait on ne la comprend pas, et qu'ensuite on découvre comment il faut la lire) (Wittgenstein 1980 : $62,64[I, \S 4])$
\end{abstract}

Privé de toute hypostatisation, c'est-à-dire - selon ce geste typique de la deuxième manière du philosophe autrichien - privé de tout recours à une dialectique du genre type/token, le langage ne devient autre qu'une physionomie. Ainsi, ce qui permet la reconnaissance d'une phrase en tant que phrase n'est que son «son » («Satzklang ») : phrase est tout ce qui résonne comme une phrase, tout ce qui est justement « saisi per l'oreille » (« den man mit dem Ohr erfassen kann / that can be taken by the ear ») :

Ce qui se grave immédiatement en nous au cours de l'usage d'un mot est la manière dont il est employé dans la structure de la phrase, la partie de son usage - pourrait-on dire - que l'on peut saisir par l'oreille (Wittgenstein $2005: 237$ [I, § 664])

\title{
Références bibliographiques
}

Archaimbault, S. (2000). Un texte fondateur pour l'étude du dialogue : De la parole dialogale (L. Jakubinskij). Histoire Épistémologie Langage, 22/1, pp. 99-115

Bakhtine, M.M. (1929). Problemy tvorcestva Dostoevskogo [Problèmes de l'œuvre de Dostoïevski]. Leningrad: Priboj [La Poétique de Dostoïevski, Paris : Seuil $1970^{1}$ (version française de la $2^{\mathrm{e}}$ édition [1963])]

Bally, Ch. (1941). Intonation et syntaxe. Cahiers Ferdinand de Saussure, 1, pp. 33-42

Bally, Ch. (1965). Linguistique générale et linguistique française [1932]. Quatrième éd. revue et corrigée, Berne : Francke

Birnbaum, H. (1984). The Syntactic Vision of A. M. Peškovskij. Russian Linguistics, 8/2, pp. 147-158

Botinis, A., Granström, B. Möbius, B. (2001), Developments and Paradigms in Intonation Research. Speech Communication, 33, pp. 263-296

Comtet, R. (2003). La classification du verbe slave comme enjeu franco-russe dans la première moitié du $\mathrm{XX}^{\mathrm{e}}$ siècle : Paul Boyer, Serge Karcevski et Antoine Meillet. Slavica occitania, 16, pp. 267-316.

Daneš, F. (1960). Sentence Intonation from a functional Point of View. Word, 16, pp. 34-45

Eichenbaum, B.M. (1924). Illjuzija skaza [L'illusion du «skaz », 1918]. Skvoz' literaturu. Sbornik statej [À travers la littérature. Recueil d'articles]. Leningrad : Academia, pp. 152-156 [réimpr. 's-Gravenhage : Mouton \& Co 1962, pp. 152-156]

Fontaine, J. (1974). Le cercle linguistique de Prague. Paris : Marne

Fougeron, I. (2012). On fait une pause ? Revue des études slaves, 82/2 [« La lettre et l'esprit : entre langue et culture. Études à la mémoire de Jean Breuillard », sous la direction de S. Viellard, L. Troubetzkoy, S. Aslanoff], pp. 429442

Godel, R. (1957). Les sources manuscrites du Cours de linguistique générale de Ferdinand de Saussure. Genève : Droz [1969²]

Graffi, G. (2001). 200 Years of Syntax. A critical Survey. Amsterdam : Benjamins

Grammont, M. (1933). Traité de phonétique. $9^{\mathrm{e}}$ éd. Paris : Delagrave

Ivanova, I. (2000). Spécificités de l'étude du dialogue dans la linguistique russe. Histoire Épistémologie Langage, $22 / 1$, pp. $117-129$

Ivanova, I. (2012). Lev Jakubinskij, une linguistique de la parole (URSS, années 1920-1930). Textes édités et présentés par I.I., traductions d'I.I et P. Sériot. Limoges : Lambert Lucas 
Jakobson, R. (1956). Serge Karcevski (August 28, 1884 - November 7, 1955). Cahiers Ferdinand de Saussure, 14, pp. 9-13 [repris dans Portraits of Linguists: A Biographical Source Book for the History of Western Linguistics, 1746-1963, éd. par Th.A. Sebeok, Bloomington : Indiana University Press 1966 ${ }^{1}$, vol. 2, pp. 493-497; une version française, par J. Breuillard, se trouve dans Karcevski (2000 : XIX-XXIII)]

Jakubinskij, L.P. (1923). O dialogiceskoj reci [De la parole dialogale]. Russkaja rec', 1, pp. 96-194

Karcevski, S.O. (1922). Classification naturelle des verbes russes. Slavia, 1, t. 2/3, pp. 242-268

Karcevski, S.O. (1923). Mécanisme des aspects des verbes russes. Slavia, 1, t. 4, pp. 497-523

Karcevski, S.O. (1925). Russkij jazyk. Č. 1 : Grammatika. [Langue russe. $1^{\text {ère }}$ partie : Grammaire]. Prague : Plamja

Karcevski, S.O. (1928). Povtoritel'nyj kurs russkogo jakyka [Précis de langue russe]. Moscou : Éditions d'État

Karcevski, S.O. (1929). Du dualisme asymétrique du signe linguistique. Travaux du Cercle linguistique de Prague, 1, pp. 89-93 [réédité dans Cahiers Ferdinand de Saussure, 14 (1956), pp. 18-24 et repris dans Karcevski (2000 : 38) dont on emploie la pagination]

Karcevski, S.O. (1931). Sur la phonologie de la phrase. Travaux du Cercle linguistique de Prague, 4, pp. 188-227 [réimprimé dans Vachek (1964: 206-251) et repris dans Karcevski (2000: 87-124) dont on emploie la pagination]

Karcevski, S.O. (1932). Autour d'un problème de morphologie. Annales Academiae scientiarum Fennicae, série B, 27, pp. 85-91 [repris dans Karcevski (2000: 77-83) dont on emploie la pagination]

Karcevski, S.O. (1933). [s.t.]. Actes du $2^{e}$ Congrès international des linguistes (Genève, 1931), Paris : Maisonneuve, pp. 114-116 [repris dans Karcevski (2000:9-11)]

Karcevski, S.O. (1936). Sur la nature de l'adverbe. Travaux du Cercle linguistique de Prague, 6, pp. 107-111 [réimprimé dans Karcevski (2000 : 247-253)]

Karcevski, S.O. (1937a). Phrase et proposition. Mélanges de linguistique et de philologie offerts à J. van Ginneken à l'occasion du soixantième anniversaire de sa naissance, Paris : Klinksieck, pp. 58-66 [repris dans Karcevski (2000 : 127-134) dont on emploie la pagination]

Karcevski, S.O. (1937b). L'idée du procès dans la langue russe. Cahiers Ferdinand de Saussure, 14, pp. 25-35 [repris dans Karcevski (2000: 65-76)]

Karcevski. S.O. (1940). Deux propositions dans une seule phrase. Études de syntaxe russe. Cahiers Ferdinand de Saussure, 14 [1956], pp. 36-52 [repris dans Karcevski (2000 : 195-210) dont on emploie la pagination ; v. aussi Karcevski (2000: 211-215)].

Karcevski, S.O. (1942). Asyndète et subordination en russe. Karcevski (2000 : 217-238) [inédit. Trad. russe publiée dans Voprosy âzykoznaniâ [Questions de linguistique], 2, 1961]

Karcevski, S.O. (2000). Inédits et introuvables. Textes rassemblés et établis par I. et G. Fougeron. Avant-propos de R. L’Hérmitte. Introduction d'I. Fougeron et J. Breuillard. Louvain : Peeters

Karcevski, S.O. (2004). Système du verbe russe. Essai de linguistique synchronique [1927]. Nouvelle édition enrichie d'inédits, commentée et préfacée par I. Fougeron, J. Breuillard et G. Fougeron, Paris : Institut d'études slaves

Lepschy, G.C. (1976). La linguistique structurale [1968]. Paris : Payot

Mathesius, V. (1911). O potenciálnosti jevů jazykových [Sur la potentialité des phénomènes linguistiques]. Věstník Královské české společnosti nauk. Tř́da filosoficko-historicko-jazykozpytná, 2, pp. 1-24 [trad. Vachek (1964 : 132)]

Mathesius, V. (1929). Zur Satzperspektive im modernen English. Archiv fu das Studium der neueren Sprachen und Literaturen, 155, pp. 202-210

Mathesius, V. (1936). Ten Years of the Prague Linguistic Circle [Deset let Pražského linguistického kroužku]. Vachek (1966 : 137-151) [version abrégée et traduite de l'original tchèque par Vachek de l'article paru dans Slovo a slovesnost, 2 (1936), pp. 137-145]

Moran, J. (1972). Wittgenstein and Russia. New Left Review, I/73, pp. 83-96 
Peškovski, A.M. (1928a). Russkij sintaksis v naucnom osveščenii $\left[1914^{1}, 1956^{7}\right]$ [Syntaxe Russe à la lumière de la science]. Moscou

Peškovski, A.M. (1928b). Intonaciâ i grammatika [Intonation et grammaire]. Izvestiâ po russkomu âzyku i slovenosti, 1, t. 2, pp. 458-476.

Pierssens, M. (1990). La dissymétrie : Saussure et Karcevski. Savoirs à l'œuvre. Essais d'épistémocritique. Lille : Presses Universitaires de Lille, pp. 89-108

Pila, M. (2011). S. Karcevskij on the asymmetrical Dualism of the linguistic Sign. Cahiers de l'ILSL 31 [« Langue(s). Langage(s). Histoire(s)», éd. par E. Velmezova »], pp. 153-166

Pospelov, N.S. (1957). Iz istorij jazykoznanija. O lingvističeskom nasledstve S. Karcevskogo [De l'histoire de la linguistique. À propos de l'héritage linguistique de S.K.]. Voprosy slavjanskogo jazykoznanija, 6, pp. 46-56

Puech, Ch. (2015) (éd.). Histoire Épistémologie Langage, $37 / 2$ [" "Faire école” en linguistique au XX siècle : l'école de Genève »]

Raynaud, S. (1990). Il circolo linguistico di Praga (1926-1939). Radici storiche e apporti teorici. Milano : Vita e Pensiero

Romashko, S.A. (2000). Vers l'analyse du dialogue en Russie. Histoire Épistémologie Langage, 22/1, pp. 83-98

Rossi, M. (1981). Rossi, M., Di Cristo, A., Hirst, D., Martin, Ph., Nishinuma, Y. L'intonation : de l'acoustique à la sémantique. Paris : Klincksieck, pp. 1-15

Rossi, M. (2000). Intonation: Past, Present, Future. Botinis, A. (ed.), Intonation: Analysis, Modeling and Technology. Dordrecht : Kluwer Academic Publishers, pp. 13-52

Saussure, F. de (1922). Cours de linguistique générale [1916]. Publié par Ch. Bally et A. Sechehaye avec la collaboration d'A. Riedlinger. Édition critique préparée par T. De Mauro. Paris : Payot [1972 $\left.{ }^{1}\right]$

Sechehaye, A. (1927). L'école genevoise de linguistique générale. Indogermanische Forschungen, 44, pp. 217-241

Ščerba, L.V. (1915). Vostočnoluiickoje narečie [Un dialecte sorabe de l'est], t. 1. Petrograd : Kollins

Steiner, W. (1976). Language as a process : Sergej Karcevskij's Semiotics of Language. Sound, Sign and Meaning. Quinquagenary of the Prague Linguistic Circle, éd. L. Matejka. Ann Harbor : The University of Michigan, pp. 291-300 [repris dans Language, Literature and Meaning I. Problems of Literary Theory, éd. J. Odmark, Amsterdam : Benjamins 1979, pp. 99-114]

Stelling-Michaud, S. (1956). Notice biographique. Cahiers Ferdinand de Saussure, 14, pp. 5-7.

Vachek, J. (1964). A Prague School Reader in Linguistics. Bloomington / Londres : Indiana University Press

Vachek, J. (1966). The Linguistic School of Prague. An Introduction to its Theory and Practice. Bloomington: Indiana University Press

Velmezova, E. (2008). L'étude des interjections à la lumière de la réception des idées saussuriennes en Russie. Bulletin of the Henry Sweet Society for the History of Linguistic Ideas, 50, pp. 45-56

Vinogradov, V.V. (1938). Sovremennyj russkij âzyk [Le russe contemporain], II. Moscou : Učpedgiz

Vsevolodskij-Gerngross, V. (1922). Teoriâ russkoj rečevoj intonacii [Théorie de l'intonation du discours russe]. St. Pétersbourg

Wittgenstein, L. (1980). Grammaire philosophique [1969]. Trad. de l'allemand et présenté par M.-A. Lescourret. Paris : Gallimard

Wittgenstein, L. (2005). Recherches philosophiques [1953]. Trad. de l'allemand par F. Dastur, M. Élie, D. Janicaud, avant-propos et apparat critique d'É. Rigal. Paris : Gallimard

\footnotetext{
${ }^{1}$ Ainsi, par exemple, Fontaine (1974 : 126) ou Graffi (2001 : 179). Les traits principaux de la recherche linguistique de la période dite «classique » du Cercle de Prague sont présentés dans Vachek (1966), Fontaine (1974), Lepschy (1976: 57-78), Raynaud (1990); sur l'École de Genève voir dernièrement Puech (2015). Au-delà de
} 
l'entreprise de récupération de l'œuvre de Karcevski menée par G. et I. Fougeron (Karcevski 2000, 2004), parmi les travaux les plus récents portant sur des points spécifiques nous rappelons Velmezova (2008) et Pila (2011). La présente étude a été réalisée dans le cadre du projet LabEx TransferS Genèse et transferts des savoirs linguistiques dirigé par Irène Fenoglio (ITEM); http://www.transfers.ens.fr/article162.html

${ }^{2}$ Trente-cinq titres, y compris deux ouvrages (Karcevski 1928 ; 2004), plus une vingtaine de comptes-rendus, parus entre 1921 et 1956. Une bibliographie complète de ses écrits est donnée dans Karcevski (2000 : 261-264).

${ }^{3}$ Ce syntagme se retrouve à maintes reprises dans l'ensemble de ses travaux; cf. par ex. Karcevski (2000: 82 [« Autour d'un problème de morphologie » (1932)], 7 [ " Du dualisme asymétrique du signe linguistique » (1929)]).

${ }^{4}$ Jakobson (1956), Stelling-Michaud (1956), Pospelov (1957), Vachek (1966 : 128), Raynaud (1990 : 151-152), Karcevski (2000 : VII-XVIII ; 2004 : XV-XXII).

${ }^{5}$ La question de la participation réelle de Karcevski aux cours des maîtres genevois reste ouverte. Pour ne citer qu'une notice d'autorité, en 1927 Karcevski est mentionné - avec Bally et Marguerite Lips (1892-1993) - parmi les élèves de Saussure dans « L'École genevoise de linguistique générale », article rédigé par A. Sechehaye (1870-1946). Par ailleurs, les aperçus biographiques mentionnés ci-dessus rapportent génériquement que Karcevski a suivi «les cours » de Saussure et Bally (et d'A. Sechehaye), alors que les registres de l'Université de Genève ne relèvent sa présence que pour un seul cours professé par Saussure (Sanskrit, 1911/1912) ainsi que pour plusieurs par Ch. Bally (Toman 1994 ; Godel 1957 : 26).

${ }^{6}$ L'ouvrage n'aborde strictement que la « syntagmatie interne » et « externe » du verbe (c'est-à-dire : dérivation et syntaxe), sans prendre en compte la «phonologie » et la « lexicologie » (voir plus loin). Des études singulières portant sur la classification verbale et sur le mécanisme des aspects, qui intégraient le projet originaire, étaient déjà parus (Karcevski 1922, 1923). Sur les apports et les enjeux de la classification du verbe russe proposée par Karcevski - qui fait preuve d'un geste théorique de grande originalité - v. Comtet (2003) et Karcevski (2004 : VII-XXII).

${ }^{7}$ Karcevski (2000 : 21-45) et Karcevski (2004 : 11-36), ce dernier rapportant un texte légèrement retouché. La première ébauche de rédaction, datée du 4 mai 1917 et assez différent de l'imprimé, est publiée également dans Karcevski (2004 : 173-180).

${ }^{8}$ Trois des huit volumes des Travaux du Cercle linguistique de Prague accueillent des articles de Karcevski (1929; $1931 ; 1936)$.

${ }^{9}$ Un résumé de cette communication paraît dans le Bulletin du Cercle linguistique de Copenhague, 6 (1939-1940, pp. 6-8), alors qu'une version tirée d'un manuscrit inédit sera publiée dans le Cahiers Ferdinand de Saussure 14 (Karcevski 1940). Une version de ce même texte qui tient compte des variantes de six manuscrits différents est publiée dans Karcevski (2000 : 195-210), avec un texte inédit portant le même intitulé (Karcevski 2000 : 211-215).

${ }^{10}$ L'article de Pospelov (1957) est centré sur l'analyse de la notion de «dualisme asymétrique » du signe comme croisement de l'homonymie et de la synonymie, notion énoncée dans Karcevski (1929). Ce principe de l'«équilibre instable » (ibid., 7), avec sa portée sémiologique générale (il est la clé qui permet l'adhésion, l'adéquation de la langue à la « situation concrète », ibid.) sera mis en œuvre dans chaque domaine de recherche abordé par Karcevski : de la syntaxe à la morphologie, du mot à la phrase, à l'intonation. La portée révolutionnaire de cette interprétation est bien reconnue par Pospelov (elle faisait « éclater de l'intérieur la conception de la linguistique statique de l'École de Genève », p. 49) et elle est répétée aussi par d'autres interprètes plus récents (Steiner 1976 ; Pierssens 1990).

${ }^{11}$ Voir seulement Saussure (1922: 20-22, 23-35), y compris le commentaire.

${ }^{12}$ De cette manière par ailleurs, Karcevski s'écarte également de ses compatriotes formalistes, qui, quant à eux, visent à dégager le profil d'une structure, gardant la forme comme pierre angulaire de toute approche théorique (littéraire d'abord, mais aussi linguistique).

${ }^{13}$ Bally (1965 [1932] : 35); voir plus loin.

${ }^{14}$ Voir Rossi (1981) pour une histoire cursive des différentes positions des linguistes du XX $\mathrm{X}^{\mathrm{e}}$ siècle vis-à-vis de la véritable question d'épistémologie linguistique posée par le traitement du phénomène de l'intonation. D'autres études prennent en compte une perspective plus large, qui implique les développements actuels des théories de la prosodie : Rossi (2000), Botinis, Granström, Möbius (2001).

${ }^{15}$ C'est à Daneš (1960) que l'on doit une reprise importante de cette même approche. 
${ }^{16}$ Karcevski (2004 : 11-18). Sa réponse à la troisième question posée aux linguistes dans le cadre du $2^{\mathrm{e}}$ Congrès international des Linguistes, à Genève en 1933 ( «Les systèmes phonologiques envisagés en eux-mêmes et dans leurs rapports avec la structure générale de la langue ») en constitue un résumé efficace (Karcevski 1933).

${ }^{17}$ « La morphologie - affirme Karcevski dans une note conçue comme explication à sa classification des verbes russes - est le système des procédés employés par la langue donnée pour révéler sur la ligne phonique les rapports projetés », Karcevski (1932:82).

${ }^{18}$ Karcevski (1931:89ss, 116ss ; 92).

${ }^{19}$ L'ouvrage de Peškovski (1928a) ne comporte aucune section entièrement consacrée à l'étude de l'intonation per se : celle-ci est pourtant l'un des paramètres principaux utilisés dans la description de chaque structure syntaxique analysée.

${ }^{20}$ Une vue d'ensemble sur la pensée syntaxique de Peškovski est donnée par Birnbaum (1984).

${ }^{21}$ L'autre est H. Bergson (Karcevski 1931 : 90).

${ }^{22}$ Karcevski (1931: 106).

${ }^{23}$ V. Fougeron (2012).

${ }^{24}$ Tous les exemples suivants sont donnés par Karcevski (1931: 103, 113 et passim).

${ }^{25}$ La phrase se poursuit dans une version manuscrite : «mené par le sujet pensant avec lui-même », Karcevski (2000: 133).

${ }^{26}$ Karcevski (1940 : 39).

${ }^{27}$ Premier essai consacré entièrement à l'examen de la forme dialogale, à ses éléments structuraux fondamentaux ainsi qu'à ses tenants psychologiques, cet article a été récemment présenté, traduit en français et annoté par I. Ivanova (2012:39-172) ; v. aussi Archaimbault (2000)

${ }^{28}$ Romashko (2000) ; v. aussi Ivanova (2000) pour les ramifications plus récentes des études.

${ }^{29}$ V. par exemple Eichenbaum (1924); Romashko (2000: 88-91). La référence rapide à Vinogradov (1938: 538), qu'on trouve à l'occasion dans Karcevski (1940:201-202), se limite à une question strictement syntaxique.

${ }^{30}$ Profondément attiré par la culture russe, et notamment par la littérature, Wittgenstein prit des cours de Russe à partir de 1934, et visita (au moins) Leningrad en Septembre 1935 (v. Moran 1972, qui fait le point sur les implications générales, y compris idéologiques, de cette fascination); cependant, toute référence à la linguistique russe (ou genevoise) est absente de l'horizon de Wittgenstein.

${ }^{31}$ «On appelle proposition tout ce qui peut être vrai ou faux », Wittgenstein (1980: 165 [I, 79]). 